\title{
Entropy-based critical reaction time for mixing-controlled reactive transport
}

\author{
Chiogna, Gabriele; Rolle, Massimo
}

Published in:

Water Resources Research

Link to article, DOI:

10.1002/2017WR020522

Publication date:

2017

Document Version

Peer reviewed version

Link back to DTU Orbit

Citation (APA):

Chiogna, G., \& Rolle, M. (2017). Entropy-based critical reaction time for mixing-controlled reactive transport. Water Resources Research, 53(8), 7488-7498. https://doi.org/10.1002/2017WR020522

\section{General rights}

Copyright and moral rights for the publications made accessible in the public portal are retained by the authors and/or other copyright owners and it is a condition of accessing publications that users recognise and abide by the legal requirements associated with these rights.

- Users may download and print one copy of any publication from the public portal for the purpose of private study or research.

- You may not further distribute the material or use it for any profit-making activity or commercial gain

- You may freely distribute the URL identifying the publication in the public portal

If you believe that this document breaches copyright please contact us providing details, and we will remove access to the work immediately and investigate your claim 


\title{
Entropy-based critical reaction time for mixing-controlled reactive transport
}

\author{
Gabriele Chiogna ${ }^{1,2^{*}(\mathbb{D})}$, Massimo Rolle ${ }^{3(\mathbb{D})}$ \\ ${ }^{1}$ Faculty of Civil, Geo and Environmental Engineering, Technical University of Munich, Arcistr. \\ 21, 80333 Munich, Germany
}

${ }^{2}$ Institute of Geography, University of Innsbruck, Innrain 52f, 6020 Innsbruck, Austria

${ }^{3}$ Department of Environmental Engineering, Technical University of Denmark, Bygningstorvet, 2800 Lyngby, Denmark

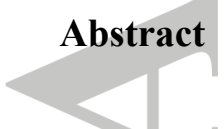

Entropy-based metrics, such as the dilution index, have been proposed to quantify dilution and reactive mixing in solute transport problems. In this work, we derive the transient advection dispersion equation for the entropy density of a reactive plume. We restrict our analysis to the case where the concentration distribution of the transported species is Gaussian and we observe that, even in case of an instantaneous complete bimolecular reaction, dilution caused by dispersive processes dominates the entropy balance at early times and results in the net increase of the entropy density of a reactive species. Successively, the entropy of the reactant decreases until it vanishes. We show the existence of a unique critical value of dilution, which corresponds to the complete consumption of one of the reactants. This critical dilution index is independent of advective and dispersive processes, and depends only on the dimensionality of the problem, on the stoichiometry of the reaction and on the initial concentrations of the reactants. Furthermore, we provide simple analytical expressions to compute the critical reaction time, i.e., the time at which the critical dilution index is reached, for selected flow configurations. Our results show that, differently from the critical dilution index, the critical reaction time depends on solute transport processes such as advection and hydrodynamic dispersion.

This article has been accepted for publication and undergone full peer review but has not been through the copyediting, typesetting, pagination and proofreading process which may lead to differences between this version and the Version of Record. Please cite this article as an 'Accepted Article', doi: 10.1002/2017WR 020522 


\section{Highlights}

- Derivation of the transient transport equation for the entropy of a reactive solute

- Definition of the concepts of critical reaction time and dilution index, and analytical solutions are proposed for their calculations

- The critical reaction time depends on mixing processes, whereas the critical dilution index does not

\section{Introduction}

The Shannon entropy has been successfully applied to describe dilution during transport of conservative tracers and it is generally expressed in terms of dilution index, which essentially quantifies the effective volume of a solute slug [Kitanidis, 1994; Cao and Kitanidis, 1998; Tartakovsky et al., 2009; Dentz et al., 2011; Rolle et al., 2013; Schneider et al., 2013; Rolle and Kitanidis, 2014; Porta et al., 2015; Ben Neriah and Paster, 2016; Boon, 2016]. A modified version of the dilution index, the flux-related dilution index, has been proposed to quantify dilution as volumetric flux carrying the solute mass flux, and has been mostly used to quantify plume dilution in the case of continuous injection [Rolle et al., 2009; Chiogna et al., 2011; Muniruzzaman et al., 2014; Cirpka et al., 2015; Ye et al., 2015a and b].

Linking the concept of dilution index with reactive mixing has been attempted by Chiogna et al., [2011 and 2012] considering steady-state flow and transport conditions; however, the entropy dynamics of conservative and reactive species has not been explored, yet, for transient transport problems. Transport and mixing exert an important control for a wide variety of subsurface reactive processes, including mineral precipitation and dissolution reactions [Molins et al. 2012; Yoon et al., 2012; Li et al., 2014], redox processes [Rolle et al., 2008, Bjerg et al., 2011] and degradation of organic contaminants at the fringe of groundwater plumes [Bauer et al., 2009; Prommer et al., 2009, Essaid et al., 2015]. For some notable cases of mixing-controlled reactions (e.g., acid-base 
reactions, aerobic degradation of some organic compounds), the reactive transport problem can be solved starting from the concentration distribution of a fictitious conservative species [e.g., De Simoni et al., 2005; Luo et al., 2008; Sanchez-Villa et al., 2007; Engdahl et al., 2013]. Such species, can be defined as the mixing ratio, representing the volumetric fraction of the source water in the mixture with the ambient solution. A procedure, based on simple algebraic equations, allows computing the reactants and products concentrations based on the distribution of the conservative mixing ratio [e.g., Cirpka and Valocchi, 2007; Cirpka et al., 2012; Chiogna and Bellin, 2013; Avesani et al., 2016].

In this work, we investigate the entropy evolution during reactive transport in porous media and we derive the transient transport equation for the entropy of a reactive solute. We focus on the case of an instantaneous complete bimolecular reaction and we show that, if the mixing ratio has a Gaussian distribution, there is a unique critical value of the dilution index, quantifying a critical amount of mixing, necessary to completely consume a reactive species. This quantity, defined as the critical dilution index, is independent of mixing processes, but depends only on the stoichiometric coefficients of the reaction, on the initial concentration of the reactants and on the dimensionality of the problem. We consider cases where the solution of the advection dispersion equation for the mixing ratio leads to a Gaussian concentration distribution, since such cases have been thoroughly examined in recent literature studies focusing on mixing [Bolster et al., 2011; de Barros et al., 2012; de Barros et al., 2015], and allow quantifying the dilution index with simple analytical solutions [Kitanidis, 1994]. We analyze three cases of mixing-controlled reactive transport in: (i) uniform flow, (ii) shear flow, (iii) complex flow including shearing, vorticity and stretching, and we derive simple analytical expressions to compute the critical reaction time, i.e., the time required to reach the critical dilution index. 


\section{Entropy transport equation}

Reactive solute transport in geophysical flows depends on the complex interplay between reaction kinetics and physical transport processes [e.g. Steefel and Lichtner, 2005; Hester et al., 2017; Li et al., 2017]. For solute transport in such systems an entropy balance equation can be derived. Here we exemplify the methodology for solute transport in saturated porous media, for which the governing advection-dispersion-reaction equation reads as [Bear, 1972]:

$$
\frac{\partial C_{i}}{\partial t}+\nabla \cdot\left(\mathbf{v} C_{i}\right)-\nabla \cdot\left(\mathbf{D} \nabla C_{i}\right)=r_{i}
$$

where $C_{i}\left[\mathrm{ML}^{-3}\right]$ is the concentration of a reactive species $i, t$ is time, $\mathbf{v}\left[\mathrm{LT}^{-1}\right]$ is the velocity vector, D $\left[\mathrm{L}^{2} \mathrm{~T}^{-1}\right]$ is the dispersion tensor and $r_{i}\left[\mathrm{ML}^{-3} \mathrm{~T}^{-1}\right]$ is the reaction term.

In order to derive the transport equation for the entropy of a reactive solute under transient conditions we follow an approach similar to the one proposed by Chiogna et al. [2012] for steadystate transport.

We start by defining the operator $\mathbf{L}$ as:

$$
\mathrm{L}=\frac{\partial}{\partial t}+\nabla \mathbf{v}-\nabla \cdot(\mathbf{D} \nabla)
$$

and the quantity $s$ as:

$$
\begin{aligned}
s=f\left(p_{i}(\mathbf{x}, t)\right) & =-p_{i}(\mathbf{x}, t) \ln \left(p_{i}(\mathbf{x}, t)\right) \\
& =-\frac{C_{i}(\mathbf{x}, t)}{\int_{V} C_{i}(\mathbf{x}, t) d V} \ln \left(\frac{C_{i}(\mathbf{x}, t)}{\int_{V} C_{i}(\mathbf{x}, t) d V}\right)
\end{aligned}
$$

where $p_{i}(\mathbf{x}, t)\left[\mathrm{L}^{-3}\right]$ is the concentration normalized by the total mass of the solute $i$, i.e., the probability distribution of the location of a tagged particle of the solute $i$ at time $t$ [Kitanidis, 1994]. For simplicity, and following the terminology used in other literature studies [e.g., Liu, 2007; Tehseen and Broadbridge, 2012], in this manuscript we denote $s$ as Shannon entropy density. 
Notice that for a reactive transport problem, $C_{i}$ satisfies the transport equation (Equation 1), and considering a non-zero reaction term, the integral $\int_{V} C_{i} d V$ is not constant in time. This term will decrease if $C_{i}$ is the concentration of a reactant that is consumed upon reaction and will increase if $C_{i}$ is the concentration of a reaction product.

Applying the operator $\mathbf{L}$ to the function $f$, assuming divergence free flow, and then using the chain rule of differentiation for the different terms, we obtain:

$$
\begin{gathered}
\frac{\partial f\left(p_{i}(\mathbf{x}, t)\right)}{\partial t}=\frac{\partial f\left(p_{i}(\mathbf{x}, t)\right)}{\partial p_{i}(\mathbf{x}, t)} \frac{\partial p_{i}(\mathbf{x}, t)}{\partial t} \\
\mathbf{v} \cdot \nabla f\left(p_{i}(\mathbf{x}, t)\right)=\frac{\partial f\left(p_{i}(\mathbf{x}, t)\right)}{\partial p_{i}(\mathbf{x}, t)} \mathbf{v} \cdot \nabla p_{i}(\mathbf{x}, t) \\
\boldsymbol{\nabla} \cdot\left(\mathbf{D} \boldsymbol{\nabla}\left(f\left(p_{i}(\mathbf{x}, t)\right)\right)\right)=\boldsymbol{\nabla} \cdot\left(\mathbf{D} \frac{\partial f\left(p_{i}(\mathbf{x}, t)\right)}{\partial p_{i}(\mathbf{x}, t)} \nabla p_{i}(\mathbf{x}, t)\right)= \\
=\frac{\partial f\left(p_{i}(\mathbf{x}, t)\right)}{\partial p_{i}(\mathbf{x}, t)} \nabla \cdot\left(\mathbf{D} \nabla p_{i}(\mathbf{x}, t)\right) \\
+\frac{\partial^{2} f\left(p_{i}(\mathbf{x}, t)\right)}{\partial\left(p_{i}(\mathbf{x}, t)\right)^{2}} \nabla\left(p_{i}(\mathbf{x}, t)\right)^{T} \mathbf{D} \nabla p_{i}(\mathbf{x}, t)
\end{gathered}
$$

Grouping the terms with the partial derivative of $f$ with respect to $p$ we obtain:

$$
\mathbf{L}\left(f\left(p_{i}(\mathbf{x}, t)\right)\right)=\frac{\partial f\left(p_{i}(\mathbf{x}, t)\right)}{\partial p_{i}(\mathbf{x}, t)}\left(\mathbf{L}\left(p_{i}(\mathbf{x}, t)\right)\right)-\frac{\partial^{2} f\left(p_{i}(\mathbf{x}, t)\right)}{\partial\left(p_{i}(\mathbf{x}, t)\right)^{2}} \nabla\left(p_{i}(\mathbf{x}, t)\right)^{T} \mathbf{D} \nabla p_{i}(\mathbf{x}, t)
$$

Using the definition of $f\left(p_{i}(\boldsymbol{x}, t)\right)$ given in Equation 3, then:

$$
\mathbf{L}\left(f\left(p_{i}(\mathbf{x}, t)\right)\right)=\frac{\partial f\left(p_{i}(\mathbf{x}, t)\right)}{\partial p_{i}(\mathbf{x}, t)}\left(\mathbf{L}\left(p_{i}(\mathbf{x}, t)\right)\right)+\frac{1}{p_{i}(\mathbf{x}, t)} \nabla\left(p_{i}(\mathbf{x}, t)\right)^{T} \mathbf{D} \nabla p_{i}(\mathbf{x}, t)
$$

in fact:

$$
\frac{\partial^{2} f\left(p_{i}(\mathbf{x}, t)\right)}{\partial\left(p_{i}(\mathbf{x}, t)\right)^{2}}=\frac{\partial^{2}-p_{i}(\mathbf{x}, t) \ln \left(p_{i}(\mathbf{x}, t)\right)}{\partial\left(p_{i}(\mathbf{x}, t)\right)^{2}}=-\frac{\partial}{\partial\left(p_{i}(\mathbf{x}, t)\right)}\left(\ln \left(p_{i}(\mathbf{x}, t)\right)+1\right)=-\frac{1}{p_{i}(\mathbf{x}, t)}
$$


The first term of the right hand side of the transport equation of the entropy density (Equation 5) entails the information about the reaction of $C_{i}$, namely:

$$
\begin{aligned}
\mathbf{L}\left(p_{i}(\mathbf{x}, t)\right)= & \frac{\partial p_{i}(\mathbf{x}, t)}{\partial C_{i}(\mathbf{x}, t)}\left(\mathbf{L}\left(C_{i}(\mathbf{x}, t)\right)\right)-\frac{\partial^{2} p_{i}(\mathbf{x}, t)}{\partial\left(C_{i}(\mathbf{x}, t)\right)^{2}} \nabla\left(C_{i}(\mathbf{x}, t)\right)^{T} \mathbf{D} \nabla C_{i}(\mathbf{x}, t)= \\
& =\frac{\partial p_{i}(\mathbf{x}, t)}{\partial C_{i}(\mathbf{x}, t)} r_{i}-\frac{\partial^{2} p_{i}(\mathbf{x}, t)}{\partial\left(C_{i}(\mathbf{x}, t)\right)^{2}} \nabla\left(C_{i}(\mathbf{x}, t)\right)^{T} \mathbf{D} \nabla C_{i}(\mathbf{x}, t)
\end{aligned}
$$

Summarizing, Equation 9 represents the transport equation for the entropy density for a reactive transport problem:

$$
\begin{aligned}
& \mathbf{L}\left(-p_{i}(\mathbf{x}, t) \ln \left(p_{i}(\mathbf{x}, t)\right)\right) \\
& =\frac{\partial p_{i}(\mathbf{x}, t) \ln \left(p_{i}(\mathbf{x}, t)\right)\left(\frac{\partial p_{i}(\mathbf{x}, t)}{\partial p_{i}(\mathbf{x}, t)} r_{i}(\mathbf{x}, t)\right.}{-} \\
& \left.-\frac{\partial^{2} p_{i}(\mathbf{x}, t)}{\partial\left(C_{i}(\mathbf{x}, t)\right)^{2}} \nabla\left(C_{i}(\mathbf{x}, t)\right)^{T} \mathbf{D} \nabla C_{i}(\mathbf{x}, t)\right)+\frac{1}{p_{i}(\mathbf{x}, t)} \nabla\left(p_{i}(\mathbf{x}, t)\right)^{T} \mathbf{D} \nabla p_{i}(\mathbf{x}, t)
\end{aligned}
$$

where we substituted Equation 8 in Equation 6, and we replaced $\mathbf{L}\left(C_{i}\right)$ with $r_{i}$, based on the governing reactive transport equation (Equation 1).

The second term of the right hand side of Equation 9 depends only on the physical process of hydrodynamic dispersion and represents a source term for the entropy density. In fact, $p$ is a probability density and therefore $p_{i}(\mathbf{x}, t) \geq 0$, the elements of $\mathbf{D}$ are all positive, and hence $\nabla\left(p_{i}(\mathbf{x}, t)\right)^{T} \mathbf{D} \nabla p_{i}(\mathbf{x}, t) \geq 0$ The first term on the right hand side of Equation 9 represents the contribution of reactive mixing and can act as the only possible sink term for the entropy. Notice also that the asymptotic behavior for $r_{i} \rightarrow 0$ is consistent with the conservative case [Kitanidis, 1994]. In fact, considering the term that is multiplied by the second derivative of $p_{i}(\mathbf{x}, t)$ with respect to $C_{i}$, and given that the probability density $p$ is defined as $p_{i}(\mathbf{x}, t)=\frac{C_{i}(\mathbf{x}, t)}{\int_{V} C_{i}(\mathbf{x}, t) d V}$, if $r_{i} \rightarrow 0$ 
the mass of the reactant tends to a constant (i.e., conservative tracer case) and hence its second derivative is zero. Therefore, the reactive mixing term vanishes since:

$$
\begin{gathered}
\lim _{r_{i} \rightarrow 0}\left(-\frac{\partial p_{i}(\mathbf{x}, t) \ln \left(p_{i}(\mathbf{x}, t)\right)}{\partial p_{i}(\mathbf{x}, t)}\left(\frac{\partial p_{i}(\mathbf{x}, t)}{\partial C_{i}(\mathbf{x}, t)} r_{i}-\frac{\partial^{2} p_{i}(\mathbf{x}, t)}{\partial\left(C_{i}(\mathbf{x}, t)\right)^{2}} \nabla\left(C_{i}(\mathbf{x}, t)\right)^{T} \mathbf{D} \nabla C_{i}(\mathbf{x}, t)\right)\right) \\
=\lim _{r_{i} \rightarrow 0}\left(-\frac{\partial p_{i}(\mathbf{x}, t) \ln \left(p_{i}(\mathbf{x}, t)\right)}{\partial p_{i}(\mathbf{x}, t)}\left(\frac{\partial p_{i}(\mathbf{x}, t)}{\partial C_{i}(\mathbf{x}, t)} r_{i}\right.\right. \\
-\underbrace{\left.\frac{\partial^{2} p_{i}(\mathbf{x}, t)}{\partial\left(C_{i}(\mathbf{x}, t)\right)^{2}} \nabla\left(C_{i}(\mathbf{x}, t)\right)^{T} \mathbf{D} \nabla C_{i}(\mathbf{x}, t)\right)}_{\rightarrow 0})=0
\end{gathered}
$$

and Equation 9 converges to the entropy transport equation proposed by Kitanidis [1994] for a conservative solute:

$$
\mathbf{L}\left(-p_{i}(\mathbf{x}, t) \ln p_{i}(\mathbf{x}, t)\right)=\frac{1}{p_{i}(\mathbf{x}, t)} \nabla\left(p_{i}(\mathbf{x}, t)\right)^{T} \mathbf{D} \nabla\left(p_{i}(\mathbf{x}, t)\right)
$$

\section{Properties of a Gaussian concentration distribution: dilution index and critical dilution} index

\subsection{Conservative transport}

We consider now the case in which the concentration distribution of a conservative solute is Gaussian. This case represents the solution of Equation 1 for an instantaneous point injection occurring at $t=0$ and at $\mathbf{x}=0$ of a conservative quantity $X$, considering constant velocity and dispersion coefficients: 


$$
X(\boldsymbol{x}, t)=\frac{1}{\operatorname{det}(\boldsymbol{k})^{1 / 2}(2 \pi)^{n / 2}} \exp \left(-\frac{1}{2}(\boldsymbol{x}-\boldsymbol{v} t)^{T} \boldsymbol{k}^{-1}(\boldsymbol{x}-\boldsymbol{v} t)\right)
$$

where $\boldsymbol{k}$ is the covariance matrix and $n$ is the dimension of the domain.

The peak, i.e. the maximum value, of a Gaussian concentration distribution is given by:

$$
X_{\text {peak }}=\frac{1}{\operatorname{det}(\boldsymbol{k})^{1 / 2}(2 \pi)^{n / 2}}
$$

The peak concentration therefore depends only on the covariance matrix of the concentration distribution and all Gaussian plumes with a given peak concentration have the same value of $\operatorname{det}(\boldsymbol{k})$

The Shanhon entropy $S$ [Shannon, 1948] is the spatial integral of the entropy density $s$ (Equation 3) and for a Gaussian distribution is [Kitanidis, 1994]:

$$
S=-\ln \left((2 \pi e)^{n / 2} \operatorname{det}(\boldsymbol{k})^{1 / 2}\right)
$$

The dilution index, defined as the exponential of Equation 14, therefore, can be expressed as a function of the peak concentration by substituting Equation 13 in Equation 14:

$$
E=(2 \pi e)^{n / 2} \frac{1}{(2 \pi)^{n / 2} X_{\text {peak }}}=\frac{e^{n / 2}}{X_{\text {peak }}}
$$

The dilution index has units of [ $\left.\mathrm{L}^{n}\right]$ where $n$ is the spatial dimension of the problem (i.e., $n=1,2$ or 3). Notice that the Gaussian distribution is the maxentropic distribution, i.e., it has the maximum entropy (and therefore the maximum dilution) for a given zero, first and second moment of the concentration distribution [Kitanidis, 1994].

\subsection{Reactive transport}

Considering a reactive transport system in which a mixing-controlled chemical reaction $\mathrm{a} A+\mathrm{b} B \rightarrow$

$\mathrm{cC}$ occurs, and assuming the same diffusive/dispersive properties of reactants and products, it is possible to compute the spatio-temporal distribution of the reacting species from the concentration of the conservative mixing ratio. For the case of an instantaneous complete bimolecular reaction 
(i.e., $C_{A} C_{B}=0$ ), the reactive species $A$ injected in a domain in which only the reactant $B$ is present, can exist as long as $X<X_{\text {crit, }}$ where $X_{c r i t}=a B_{a m b} /\left(a B_{a m b}+b A_{\text {in }}\right), a$ and $b$ are the stoichiometric coefficients of $A$ and $B$ respectively, $B_{a m b}$ is the ambient concentration of $B$, and $A_{\text {in }}$ is the inlet concentration of $A$ [Cirpka and Valocchi, 2007]. Therefore, when the peak concentration of the mixing ratio reaches the critical value, $X_{\text {peak }}=X_{\text {crit, }}$ the reactant $A$ is completely consumed and no reaction can occur anymore.

Considering the entropy for this reactive transport problem, we can interpret Equation 15 as the amount of dilution required to completely consume reactant $A$, i.e. what we define as the critical dilution index $E_{\text {crit }}$. This quantity only depends on the dimensionality of the system, on the stoichiometry of the reaction and on the initial concentrations of the reactants $A$ and $B$. In fact, substituting the definition of the critical mixing ratio $X_{c r i t}$ in Equation 15 we obtain:

$$
E_{\text {crit }}=\frac{e^{n / 2}}{X_{\text {crit }}}=e^{n / 2} \frac{b A_{\text {in }}+a B_{a m b}}{a B_{a m b}}
$$

We can now define a new important quantity, the critical reaction time $t_{c r i t}$, which is the time at which the entropy reaches the value of the critical dilution index, $E\left(t_{c r i t}\right)=E_{c r i t}$, and the reactant $A$ is completely consumed. As will be shown in the following examples, the critical reaction time is influenced by the complexity of the flow field and by the matrix variance describing the mixing processes.

Notice that the critical dilution index is computed based on the maxentropic Gaussian distribution. Therefore, the critical reaction time $t_{c r i t}$ is the time required to reach a given peak concentration for an optimally mixed system.

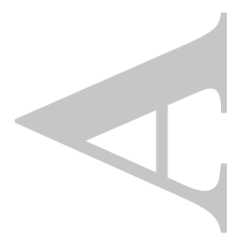




\section{Illustrative examples for the critical dilution index and the critical reaction time}

In this section we consider three illustrative examples of mixing-controlled reactive transport in two dimensional flow fields with increasing complexity, for which we analyze the entropy evolution and we derive analytical expressions to compute the critical dilution index and the critical reaction time.

We consider a uniform flow field, a shear flow field and a complex flow field. The latter two cases have been described by Bolster et al. [2011] and de Barros et al. [2012] and the reader is redirected to those work for more details. Focusing on the entropy analysis for conservative and mixingcontrolled reactive transport we have computed the concentration distribution of a conservative tracer introduced instantaneously (Dirac pulse) in two-dimensional unbounded domains. The concentration distribution of the reactive species is computed following the mixing ratio approach of Cirpka and Valocchi [2007]. The analytical solutions for the concentration distributions in the uniform, shear flow and complex topology cases are provided in the studies of Kitanidis [1994], Bolster et al., [2011] and de Barros et al., [2012], respectively.

\subsection{Uniform flow}

As a first case, we consider uniform flow in an infinite homogeneous porous medium. Under these conditions, the peak concentration of a Dirac pulse for a conservative tracer decreases with time since $\boldsymbol{k}=2 \mathrm{Dt}$ [Kitanidis, 1994]:

$$
X_{\text {peak }}=(2 \pi t)^{-n / 2} \operatorname{det}(2 \mathbf{D})^{-1 / 2}
$$

Therefore, considering $X_{\text {peak }}=X_{\text {crit }}$, we can define this critical reaction time as:

$$
t_{\text {crit }}=\left((2 \pi)^{n / 2} X_{\text {crit }} \operatorname{det}(2 \mathbf{D})^{1 / 2}\right)^{-2 / n}
$$

The dilution index is a correct measure of plume dilution, which "scales as the peak concentration" [Kitanidis, 1994]. In the case of a Dirac pulse injection in a uniform flow field, the dilution index can be computed as: 


$$
E(t)=(2 \pi e t)^{n / 2} \operatorname{det}(2 \mathbf{D})^{1 / 2}
$$

Substituting now the critical reaction time as defined in Equation (18) into Equation (19), we can show that the value of the dilution index at $t=t_{c r i t}$ is independent from transport parameters such as the flow velocity and the dispersion coefficient, but depends only on the stoichiometry of the reaction and on the dimensionality of the problem:

$$
E\left(t_{\text {crit }}\right)=\left((2 \pi)^{n / 2} \exp (n / 2) \operatorname{det}(2 \mathbf{D})^{1 / 2}\right)\left((2 \pi)^{n / 2} X_{\text {crit }} \operatorname{det}(2 \mathbf{D})^{1 / 2}\right)^{-1}=\frac{\exp (n / 2)}{X_{\text {crit }}}
$$

This is consistent with the general result shown in the previous section (Equation 16).

Always considering the case of the instantaneous release of a solute in an infinite homogeneous domain, we now analyze the time evolution of the dilution index for both a conservative and a reactive species. Figure 1 illustrates the temporal dynamics of the entropy density for a reactive solute (Equation 9) and shows the comparison between the dilution index for conservative (black lines) and reactive (blue lines) species, considering different values for $X_{\text {crit }}$ and $D$ (for the sake of simplicity the tensor $\mathbf{D}$ is considered isotropic). Independently of the values of $X_{\text {crit }}$ and $D$ used in the four examples shown, we can observe that the shapes of the curves describing the time evolution of the entropy of the conservative and reactive solutes are the same. The entropy of the reactive solute is always framed between the entropy of the conservative species and the line $t=t_{\text {crit }}$. Whereas the entropy of a conservative solute increases linearly with time, the entropy of a reactive species shows a non-monotonic temporal behavior, as expected considering the right hand side terms in Equation 9. This is due to the opposite contribution of the mixing and reactive terms in the entropy balance of a reactant (Equation 9). At early times, the entropy of the reactant increases following the trend of the conservative case. This result, obtained for the case of uniform flow, is notable since it shows that at such early times, even in the case of instantaneous reaction kinetics, physical mixing processes clearly dominate the entropy balance. The dilution index trend for a reactant reaches a maximum and then starts decreasing when the reactive processes become 
effective enough to reduce the entropy of the reactive solute until $t=t_{c r i t}$. At that time the reactant is completely consumed, its concentration becomes 0 everywhere in the domain, and its dilution index becomes unity. Increasing values of $D$ translates the frame parallel to the time axis to the left. This means that for the same value of $E_{c r i t}$, we obtain smaller critical reaction times as $D$ increases. An increase in $X_{\text {crit }}$ translates the frame along the line of the entropy for a conservative solute towards lower values of dilution.

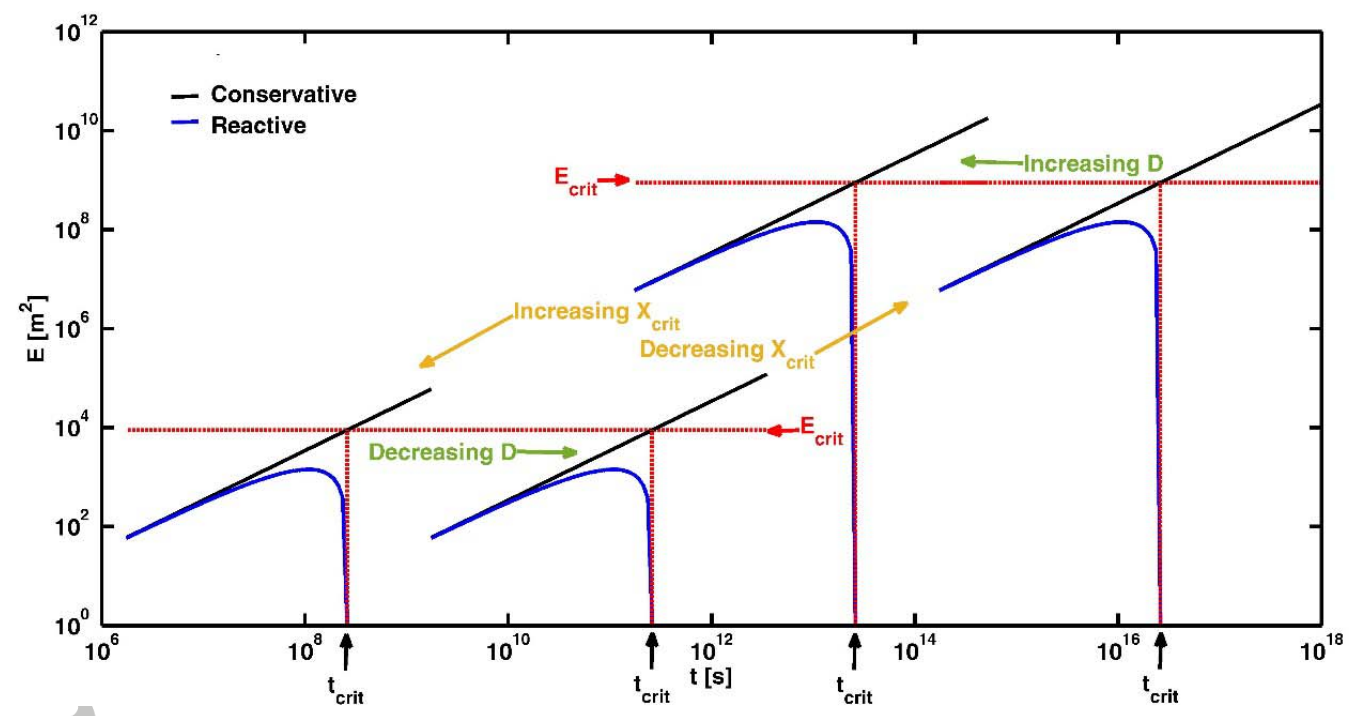

Figure 1. Relation between critical reaction time and critical dilution index in the uniform flow case for different $X_{\text {crit }}$ and $D$ values. The applied values of $X_{\text {crit }}$ are $1 \times 10^{-9}$ and $1 \times 10^{-4}$ (top and bottom curves, respectively) and of $D$ are $1 \times 10^{-9} \mathrm{~m}^{2} \mathrm{~s}^{-1}$ and $1 \times 10^{-6} \mathrm{~m}^{2} \mathrm{~s}^{-1}$ (right and left curves, respectively).

\subsection{Shear flow}

The effects of shear flow on plume deformation, conservative and mixing-controlled reactive transport have been investigated in a number of recent contributions [e.g., Bolster et al., 2011; Paster et al., 2015, Bandopadhyay et al., 2017]. The work of Bolster et al. [2011] has shown that the concentration profile in a two-dimensional (i.e., in this case $n=2$ ) shear flow computed in a

Lagrangian framework is also Gaussian. In this case, however, the variance of the Gaussian 
distribution does not depend only on the local dispersion properties as in the uniform flow case, but also on the shear rate $\alpha\left[\mathrm{T}^{-1}\right]$ :

$$
\operatorname{det}(\boldsymbol{\kappa}(t))=\left(2 D_{1} t+\frac{2}{3} D_{2} \alpha^{2} t^{3}\right)\left(2 D_{2} t\right)-\left(\alpha D_{2} t^{2}\right)^{2}=4 D_{1} D_{2} t^{2}+\frac{1}{3} D_{2}^{2} \alpha^{2} t^{4}
$$

where $D_{1}\left[\mathrm{~L}^{2} \mathrm{~T}^{-1}\right]$ is the dispersion coefficient in the main flow direction and $D_{2}\left[\mathrm{~L}^{2} \mathrm{~T}^{-1}\right]$ is the dispersion coefficient in the transverse direction. Considering that the critical dilution index is constant and independent of the mixing mechanisms, the critical reaction time can be computed as follows:

$$
E\left(t_{\text {crit }}\right)=\frac{e}{X_{\text {crit }}}=2 \pi e \sqrt{4 D_{1} D_{2} t_{c r i t}^{2}+\frac{1}{3} D_{2}^{2} \alpha^{2} t_{c r i t}^{4}} \rightarrow t_{\text {crit }}=\sqrt{\frac{-b+\sqrt{b^{2}-4 a c}}{2 a}}
$$

where $a=1, b=12 \mathrm{D}_{1} /\left(\mathrm{D}_{2} \alpha^{2}\right)$ and $c=3 /\left(2 \pi X_{\text {peak }} \mathrm{D}_{2} \alpha\right)^{2}$.

\subsection{Complex flow topology}

The impact of flow topology on plume stretching, folding and mixing has been studied at different scales in both 2-D and 3-D domains [e.g., Weeks and Sposito, 1998; Sposito, 2001; De Dreuzy et al., 2012; Piscopo et al., 2013; Chiogna et al., 2015; Crevacore et al. 2016; Lester et al., 2016]. Here we consider the work of de Barros et al., [2012], who investigated the dependence of the dilution index on the topology of the flow field in a Lagrangian framework. Despite the complexity of the considered two-dimensional flow field, also in that case it is possible to represent the concentration as a Gaussian distribution. Notice that, as discussed in de Barros et al. [2012], the validity of their solution for the concentration in a moving coordinate system is obtained assuming times smaller than the diffusion time scale, while the results for the covariance matrix $\boldsymbol{\kappa}$ are valid for times smaller than the advection time scale. The covariance of the Gaussian plume is given by: 


$$
\begin{aligned}
\operatorname{det}(\boldsymbol{\kappa}(t))= & \frac{4 D^{2}}{\theta^{6}}\left\{\left[\left(\gamma^{2}+\xi^{2}-\xi \omega\right) \sinh (\theta t)+\theta \gamma \cosh (\theta t)\right.\right. \\
& -\theta(\gamma-(\xi-\omega) \omega t)]\left[\left(\gamma^{2}+\xi^{2}+\xi \omega\right) \sinh (\theta t)-\theta \gamma \cosh (\theta t)\right. \\
& \left.+\theta(\gamma-(\xi+\omega) \omega t)]-[\gamma \omega \sinh (\theta t)+\theta \xi \cosh (\theta t)-\theta(\xi+\gamma \omega t)]^{2}\right\}
\end{aligned}
$$

where $\gamma$ is the stretching deformation, $\xi$ is shear deformation, $\omega$ is the vorticity and $\theta$ is the square root of the Okubo-Weiss parameter [Okubo, 1970; Weiss, 1991].

Again, the critical dilution index is constant and independent of the mixing properties of the system, and reads as:

$$
E\left(t_{\text {crit }}\right)=\frac{e}{X_{\text {crit }}}=(2 \pi e) \operatorname{det}\left(\boldsymbol{\kappa}\left(t_{\text {crit }}\right)\right)^{1 / 2}
$$

In this case, it is not possible to provide an explicit solution for $t_{c r i t}$, but Equation 23 can substituted into Equation 24, which can be solved iteratively for the critical reaction time.

\section{Results and discussion}

Figure 2 shows the concentration distribution and plume dilution for the cases of solute transport in uniform flow, shear flow and complex flow topology fields. All cases refer to infinite twodimensional domains and the results are visualized for a $35 \mathrm{~m} \times 6 \mathrm{~m}$ setup (Figure 2). For the cases

of shear flow (Figure 2C and 2D) and of flow with complex topology (Figure 2E and 2F), we also show the results which would be obtained in the limiting case of uniform flow; this allow appreciating the effect of mixing enhancement driven by the advective flow field. Table 1 summarizes the parameters used for the different examples shown in Figure 2. 
Table 1. Values of the parameters used for the simulations shown in Figure 2.

\begin{tabular}{lcc}
\multicolumn{1}{c}{ Parameter } & Symbol & Value \\
\hline Isotropic dispersion coefficient $\left[\mathrm{m}^{2} / \mathrm{s}\right]$ & $D$ & $1.0 \times 10^{-9}$ \\
Velocity $[\mathrm{m} / \mathrm{s}]$ & $v$ & $1.2 \times 10^{-7}$ \\
Shear rate $[1 / \mathrm{s}]$ & $\alpha$ & $5.5 \times 10^{-8}$ \\
Stretching deformation $[1 / \mathrm{s}]$ & $\gamma$ & $5.5 \times 10^{-8}$ \\
Shear deformation $[1 / \mathrm{s}]$ & $\xi$ & $5.5 \times 10^{-8}$ \\
Vorticity $[1 / \mathrm{s}]$ & $\omega$ & $8.0 \times 10^{-8}$ \\
\hline
\end{tabular}

Figure 2A shows the concentration distribution for a conservative solute transported in a uniform flow field for $t=t_{c r i t}$. We set $X_{c r i t}=0.5$ and we plot, in Figure 2B, the temporal increase of the plume dilution. In particular, we observe that at time $t=t_{\text {crit }}$ (vertical black dashed line) the value of the dilution index is equal to the value of the critical dilution index (horizontal red dashed line). This means that a reactive plume would be completely consumed after a time equal to $t_{\text {crit }}\left(1.6 \times 10^{8} \mathrm{~s}\right)$.

We consider now two limiting cases [Bolster et al., 2011] for the shear flow example. The concentration distribution is shown in Figure 2C for the base case (i.e., rotated ellipsoid obtained with $\alpha=5.5 \times 10^{-8} 1 / \mathrm{s}$ ), as well as for a second case, in which the shear rate is set to a very small value (i.e., $\alpha=1 \times 10^{-11} 1 / \mathrm{s}$ ), resulting in the same concentration distribution as in the uniform flow field. Figure 2D illustrates the temporal evolution of dilution and shows that, in presence of significant shearing $\left(\alpha=5.5 \times 10^{-8} 1 / \mathrm{s}\right)$, plume dilution increases faster than in the uniform case. We can also observe how the rotation of the plume leads to a significant reduction of the critical reaction time $\left(t_{c r i}=9.4 \times 10^{7} \mathrm{~s}\right.$, blue dotted line $)$ in comparison to the uniform flow field case. In fact, if $\alpha$ is very small (i.e., $\alpha=1 \times 10^{-11} 1 / \mathrm{s}$ ), the plume vanishes at the same time as in the uniform flow field case (vertical black dashed line in Figure 2D). However, both plumes have the same critical dilution index, $E_{\text {crit }}$ (horizontal red dashed line). 
Similar findings were obtained in the example with complex flow topology. The tilted and elongated ellipsoid in Figure 2E corresponds to the plume undergoing stretching, vorticity and shear deformation with the parameters listed in Table 1, whereas the second plume was obtained with larger values of vorticity (i.e., $\omega=5 \times 10^{-4} 1 / \mathrm{s}$ ), while keeping the same stretching and shear deformation and approaches the concentration distribution in the uniform flow field. This is due to the fact that rapid rotation of the plume indeed prevents its persistent deformation in a preferential direction. We can observe that with the chosen set of parameters the conservative tracer reaches a peak concentration equal to $X_{c r i t}$ and the dilution reaches its critical value at an earlier critical reaction time $\left(t_{c r i t}=8 \times 10^{7} \mathrm{~s}\right.$, magenta dashed line in Figure $\left.2 \mathrm{~F}\right)$, which is approximately half the value for the uniform flow case. In the case of large vorticity in comparison to shear deformation and stretching, the critical reaction time approaches the value of the uniform field (black dashed line in Figure 2F).
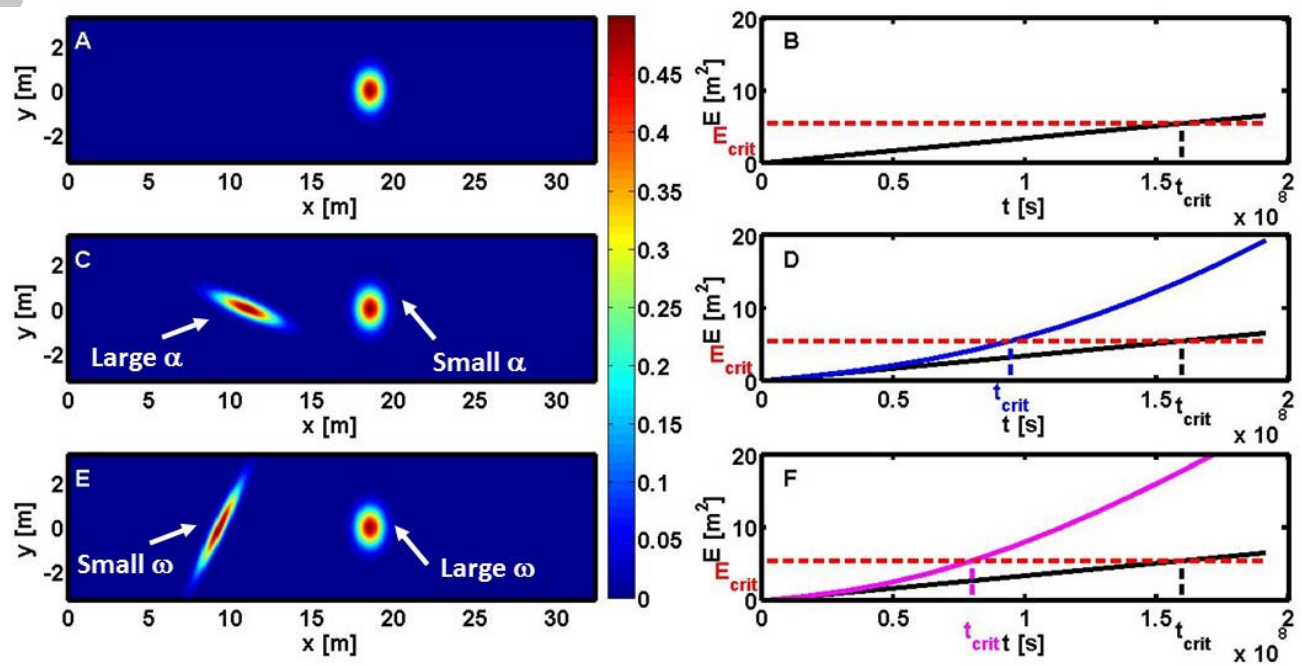

Figure 2. Concentration distribution of a conservative tracer injected in a uniform flow (A), shear flow (C) and complex flow topology (E) fields. All plumes are represented at $t=t_{\text {crit }}$. Panels B, D and $\mathrm{F}$ show the dilution index and the value of the critical dilution index (horizontal red dashed line) reached at different critical reaction times (vertical dashed lines).

It is also interesting to examine the dependence of the critical reaction time on the critical mixing ratio and, thus, on the stoichiometry and initial concentration of the reactants. Decreasing trends of 
$t_{\text {crit }}$ with increasing $X_{\text {crit }}$ have been found for all three illustrative examples as shown in Figure 3A and $3 \mathrm{C}$.

To better appreciate the effect of the advective flow field on mixing enhancement, we compute the ratio between the critical reaction time for the shear flow and the complex flow topology, respectively, and the critical reaction time for the uniform flow field case. We can observe (Figure 3B and 3D) that the larger the value of $X_{\text {crit }}$, the less effective is mixing enhancement driven by the advective properties of the flow field (i.e., the ratio between the critical reaction times approaches 1). This means that the larger the initial concentration of the injected compound $\mathrm{A}$ is (with respect to the ambient concentration of B), the more effective is mixing enhancement driven by advection. Moreover, the ratio between the critical reaction times depends on the parameters of the flow fields: it increases with decreasing shear rate in case of shear flow, while it increases with increasing vorticity in case of the complex flow field. In fact, the shear flow converges to a uniform flow by reducing the shear rate, while the complex flow field converges to the uniform flow field by increasing vorticity.
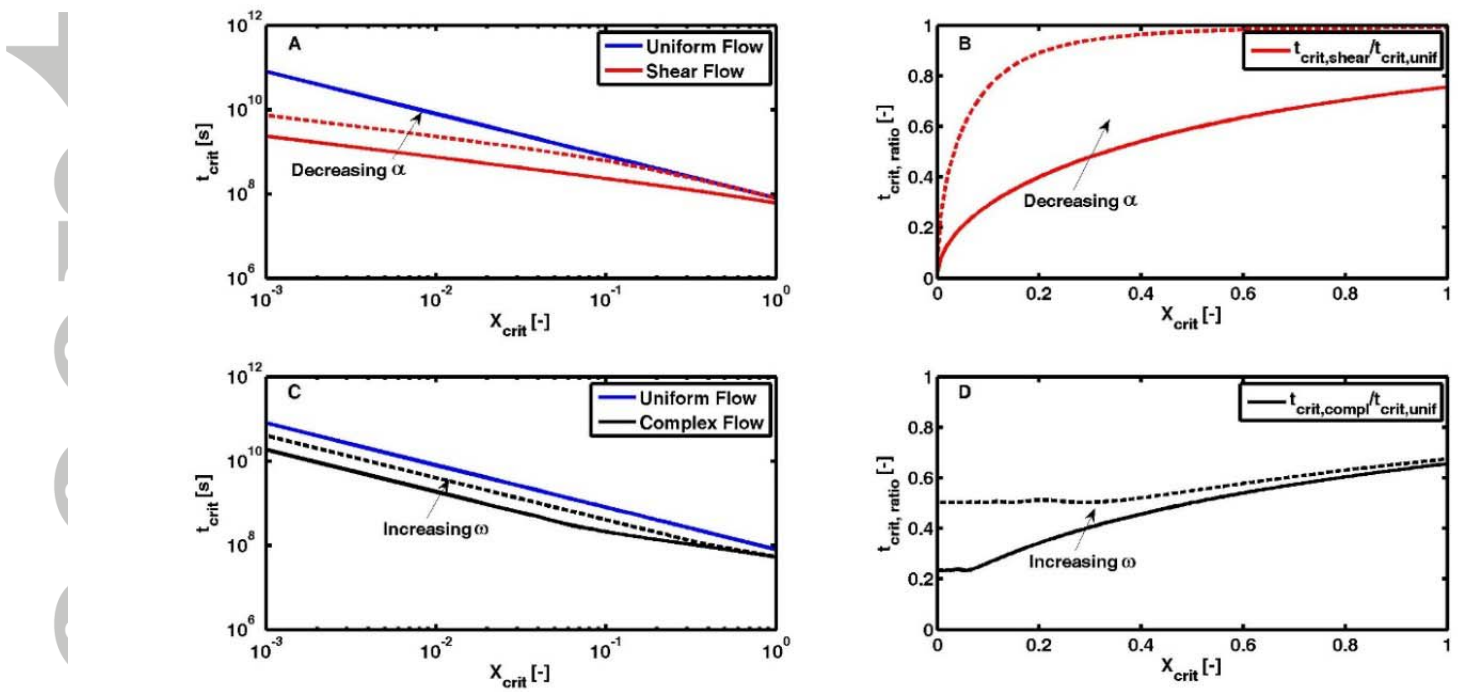

Figure 3. Relation between the critical reaction time and the critical mixing ratio for uniform flow (blue line), shear flow (red line, Panel A) and complex flow topology (black line, Panel C). Ratio between the critical reaction time in the shear flow field and in the uniform flow field (Panel B), and between the critical reaction time in the complex flow field and in the uniform flow field (Panel C), as a function of the critical mixing ratio. 


\section{Concluding remarks}

This work has investigated the entropy evolution for mixing-controlled reactive transport. The transient entropy density transport equation derived for a reactive species allowed identifying dilution terms that provide a positive contribution and act as entropy sources, as well as reactive mixing terms that act as sinks in the entropy balance. Despite this work only considered Gaussian plumes and instantaneous complete bimolecular reactions, the proposed approach and the transient entropy density transport equation can be used to investigate the temporal evolution of the dilution for reactive species in different flow fields and in case of more complex reactions (e.g., biologically mediated, reversible bimolecular equilibrium reactions etc.). Moreover, quantities like the critical dilution index and the critical reaction time have been computed for maxentropic plumes and idealized reaction kinetics. We argue, therefore, that they could both represent lower limits for more complex situations (i.e., incomplete mixing and incomplete or slow reactions).

An interesting outcome of the analysis was that, even in the case of instantaneous complete bimolecular reaction, dilution processes dominate over reaction at early time. We focused on cases

in which the solute concentration is Gaussian. Under these conditions, we have shown that it is possible to find simple analytical solutions that relate the entropy of conservative and reactive plumes with the peak concentrations. Three examples have been selected to illustrate the concepts of critical dilution index and critical reaction time. The critical dilution index quantifies the value of conservative dilution at which a transient reactant plume is completely consumed. It does not depend on mixing processes but only on the dimensionality of the problem and on the stoichiometry and initial concentration of the reactants. The newly introduced concept of the entropy-based critical reaction time, represents the time at which the critical value of dilution is reached and the reactant plume vanishes. Both the critical reaction time and the critical dilution index have been computed analytically only for Gaussian plumes. The applicability of these concepts to nonGaussian plumes has still to be tested. Moreover, the effects of incomplete mixing and spatial 
fluctuations in concentration fields on reactive solute transport [e.g., Tartakovsky et al., 2012; Chiogna and Bellin 2013] have not been explicitly considered in this work, but deserve future investigation.

The analysis provided in this study offers interesting insights for the characterization of dilution and its interaction with reactive processes in different geophysical flows. Besides the simple analytical cases illustrated in this study, the analysis of the critical dilution index and the critical reaction time can be applied for mixing-controlled reactive transport in numerical simulation in complex twodimensional and three-dimensional flow fields. Interesting outcomes, shading light on the interplay between mixing and reactions, are envisioned for mixing-controlled reactive transport in different heterogeneous and anisotropic media, for Fickian and anomalous transport.

\section{Acknowledgments}

This is a theoretical work and does not contain any data. The authors acknowledge the support of the German Research Foundation (DFG, Grant RO 4169/3-1). G.C. also acknowledges the support of the Stiftungsfonds für Umweltökonomie und Nachhaltigkeit GmbH (SUN). Constructive comments from AE Alberto Bellin, Jian Luo, and two anonymous reviewers helped improving the quality of the manuscript.

\section{References}

Avesani, D., P. Herrera, G. Chiogna, A. Bellin, and M. Dumbser (2015), Smooth Particle Hydrodynamics with nonlinear Moving-Least-Squares WENO reconstruction to model anisotropic dispersion in porous media. Adv. Water Resour., 80, 43-59, doi: 10.1016/j.advwatres.2015.03.007.

Bandopadhyay, A., T. Le Borgne, Y. Meheust and M. Dentz (2017), Enhanced reaction kinetics and reactive mixing scale dynamics in mixing fronts under shear flow arbitrary Damkohler numbers, Adv. Water Resour., 100, 78-95, doi: 10.1016/j.advwatres.2016.12.008. 
Bauer, R.D., M. Rolle, P. Kurzinger, P. Grathwohl, R.U. Meckenstock, and C. Griebler (2009), Two-dimensional flow-through microcosms - Versatile test systems to study biodegradation processes in porous aquifers. J. Hydrol., 369, 284-295, doi: 10.1016/j.jhydrol.2009.02.037.

Bear, J. (1972), Dynamics of Fluids in Porous Media, Dover, New York.

Ben Neriah, A. and A. Paster (2016). Effect of temporal changes in air injection rate on air sparging performance groundwater remediation, Groundwater, 54, 851-860, doi: 10.1111/gwat.12428.

Bjerg, P.L., N. Tuxen, L.A. Reitzel, H.-J. Albrechtsen, and P. Kjeldsen (2011), Natural attenuation processes in landfill leachate plumes at three danish sites. Groundwater, 49, 688-705, doi: 10.1111/j,1745-6584.2009.00613.x.

Bolster, D., M. Dentz, and T. Le Borgne (2011), Hypermixing in linear shear flow, Water Resour. Res., 47, W09602, doi:10.1029/2011WR010737.

Boon, M., B. Bijeljic, B. Niu and S. Krevor (2016), Observations of 3-D transverse dispersion and dilution in natural consolidated rock by X-ray tomography, Adv. Water Resour, 96, 266-281, doi: 10.1016/j.advwatres.2016.07.020.

Cao, J., and P. K. Kitanidis (1998), Pore-scale dilution of conservative solutes: An example, Water Resour. Res., 34(8), 1941-1949, doi:10.1029/98WR01468.

Chiogna, G., O.A. Cirpka, P. Grathwohl, and M. Rolle, (2011), Transverse mixing of conservative and reactive tracers in porous media: quantification through the concepts of flux-related and critical dilution indices, Water Resour. Res., 47, W02505, doi:10.1029/2010WR009608.

Chiogna, G., and A. Bellin (2013), Analytical solution for reactive solute transport considering incomplete mixing within a reference elementary volume, Water Resour. Res., 49(5), 2589-2600, doi: $10.1002 /$ wrer.20200.

Chiogna, G., D. L. Hochstetler, A. Bellin, P. K. Kitanidis and M. Rolle (2012), Mixing, entropy and reactive solute transport, Geophys. Res. Lett., 39(20), doi: 10.1029/2012GL053295.

Chiogna, G., O. A. Cirpka, M. Rolle, and A. Bellin (2015), Helical flow in three-dimensional nonstationary anisotropic heterogeneous porous media, Water Resour. Res., 51, doi:10.1002/2014WR015330. 
Cirpka, O.A., and A.J. Valocchi (2007), Two-dimensional concentration distribution for mixingcontrolled bioreactive transport in steady state, Adv. Water Resour., 30 (6-7), 1668-1679, doi: 10.1016/j.advwatres.2006.05.022.

Cirpka, O.A., M. Rolle, G. Chiogna, F.P.J. de Barros and W. Nowak (2012). Stochastic evaluation of mixing-controlled steady-state plume lengths in two-dimensional heterogeneous domains, $J$. Contam. Hydrol., 138-139, 22-39, doi:10.1016/j.jconhyd.2012.05.007.

Cirpka, O. A., G. Chiogna, M. Rolle, and A. Bellin (2015), Transverse mixing in three-dimensional nonstationary anisotropic heterogeneous porous media, Water Resour. Res., 51, doi:10.1002/2014WR015331.

Crevacore, E., T. Tosco, R. Sethi, G. Boccardo and D. Marchisio (2016), Recirculation zones induce non-Fickian transport in three-dimensional periodic porous media, Phys. Rev. E, 94, 053118, doi: 10.1103/PhysRevE.94.053118.

de Barros, F. P. J., M. Dentz, J. Koch, and W. Nowak (2012), Flow topology and scalar mixing in spatially heterogeneous flow fields, Geophys. Res. Lett., 39, L08404,doi:10.1029/2012GL051302.

de Barros, F. P. J., A. Fiori, F. Boso, and A. Bellin (2015), A theoretical framework for modeling dilution enhancement of non-reactive solutes in heterogeneous porous media, J. Contam. Hydrol., 175, 72-83, doi: 10.1016/j.jconhyd.2015.01.004.

de Dreuzy, J. R., J. Carrera, M. Dentz, and T. Le Borgne (2012), Time evolution of mixing in heterogeneous porous media, Water Resour. Res., 48, W06511, doi:10.1029/2011WR011360.

Dentz, M., T. Le Borgne, A. Englert, and B. Bijeljic (2011), Mixing, spreading and reaction in heterogeneous media: A brief review, J. Contam. Hydrol., 120-121, 1-17, doi:10.1016/j.jconhyd.2010.05.002.

De Simoni, M., J. Carrera, X. Sanchez-Vila, and A. Guadagnini (2005), A procedure for the solution of multi-component reactive transport problems, Water. Resour. Res., 41, W11410, doi:10.1029/2005WR004056.

Engdahl, N.B., T.R. Ginn, and G.E. Fogg (2013), Scalar dissipation rates in non-conservative transport systems, J. Contam. Hydrol., 149, 46-60, doi: 10.1016/j.jconhyd.2013.03.003. 
Essaid, H.I., B.A. Bekins, and I.M. Cozzarelli (2015), Organic contaminant transport and fate in the subsurface: Evolution of knowledge and understanding, Water. Resour. Res., 51, doi:10.1002/2015WR017121.

Hester, E.T., M. Bayani Cardenas, R. Haggerty, and S.V. Apte (2017), The importance and challenge of hyporheic mixing, Water Resour. Res., 53, doi:10.1002/2016WR020005.

Kitanidis, P.K. (1994), The concept of dilution index, Water Resour. Res., 30 (7), 2011-2026, doi: 10.1029/94WR00762.

Lester, D. R., M. Dentz and T. Le Borgne (2016), Chaotic mixing in three-dimensional porous media, J. Fluid Mech., 803, 144-174.

Li, L., F. Salehikhoo, S.L. Brantley, and P. Heidari (2014), Spatial zonation limits magnesite dissolution in porous media, Geochim. Cosmochim. Acta, 126, 555-573, doi: 10.1016/j.gca.2013.10.051.

Li, L. et al. (2017), Expanding the role of reactive transport models in critical zone processes, Earth-Sciences Reviews, 165, 280-301, doi: 10.1016/j.earscirev.2016.09.001.

Liu, S, (2007), On the relationship between densities of Shannon entropy and Fisher information for atoms and molecules, J. Chem. Phys., 126, 191107, doi: 10.1063/1.2741244.

Luo, J., M. Dentz, J. Carrera, and P. Kitanidis (2008), Effective reaction parameters for mixing controlled reactions in heterogeneous media, Water Resour. Res., 44(2), doi: 10.1029/2006WR005658.

Molins, S., D. Trebotich, C. I. Steefel, and C. P. Shen (2012), An investigation of the effect of pore scale flow on average geochemical reaction rates using direct numerical simulation, Water Resour. Res., 48. W03527, doi: 10.1029/2011WR011404.

Muniruzzaman, M., C.M. Haberer, P. Grathwohl, and M. Rolle (2014), Multicomponent ionic dispersion during transport of electrolytes in heterogeneous porous media: Experiments and modelbased interpretation, Geochim. Cosmochim. Acta, 141, 656-669, doi: 10.1016/j.gca.2014.06.020.

Okubo, A. (1970), Horizontal dispersion of floatable particles in the vicinity of velocity singularities such as convergences, Deep Sea Res. Oceanogr. Abstr., 17, 445-454, doi: 10.1016/0011-7471(70)90059-8. 
Paster, A., T. Aquino, and D. Bolster (2015), Incomplete mixing and reactions in laminar shear flow, Phys. Rev. E 92, 012922, doi: 10.1103/PhysRevE.92.012922.

Piscopo, A. N., R. M. Neupauer, and D. C. Mays (2013), Engineered injection and extraction to enhance reaction for improved in situ remediation, Water Resour. Res., 49, 3618-3625, doi:10.1002/wrcr.20209.

Porta, G. M., B. Bijeljic, M. J. Blunt, and A. Guadagnini (2015), Continuum-scale characterization of solute transport based on pore-scale velocity distributions. Geophys. Res. Lett., 42(18), 75377545, doi: 10.1002/2015GL065423.

Prommer, H., B. Anneser, M. Rolle, F. Einsiedl, and C. Griebler, (2009), Biogeochemical and isotopic gradients in a BTEX/PAH contaminant plume: Model-based interpretation of a highresolution field data set, Environ. Sci. Technol., 43, 8206-8212, doi:10.1021/es901142a

Rolle, M., T.P. Clement, R. Sethi, and A. Di Molfetta (2008), A kinetic approach for simulating redox-controlled fringe and core biodegradation processes in groundwater: model development and application to a landfill site in Piedmont, Italy. Hydrol. Process. 22, 4905-4921, doi: 10.1002/hyp.7113.

Rolle, M., C. Eberhardt, G. Chiogna, O.A. Cirpka, and P. Grathwohl (2009), Enhancement of dilution and transverse reactive mixing in porous media: Experiments and model-based interpretation, J. Contam. Hydrol., 110, 130-142, doi: 10.1016/j.jconhyd.2009.10.003.

Rolle, M., G. Chiogna, D.L. Hochstetler, and P.K. Kitanidis (2013), On the importance of diffusion and compound-specific mixing for groundwater transport: An investigation from pore to field scale, J. Contam. Hydrol., 153, 51-68, DOI: 10.1016/ j.jconhyd.2013.07.006.

Rolle, M., and P.K. Kitanidis (2014), Effects of compound-specific dilution on transient transport and solute breakthrough: A pore-scale analysis, Adv. Water Resour., 71, 186-199, DOI: 10.1016/j.advwatres.2014.06.012.

Sanchez-Vila, X., M. Dentz, and L. D. Donado (2007), Transport-controlled reaction rates under local non-equilibrium conditions, Geophys. Res. Lett., 34, L10404, doi:10.1029/2007GL029410.

Schneider, B., A. Paster, and D. Bolster (2013), A numerical investigation of mixing and spreading across an angled discontinuity, Adv. Water Resour., 62, 280-291, doi 10.1016/j.advwatres.2013.09.003. 
Sposito, G. (2001), Topological groundwater hydrodynamics, Adv. Water Resour., 24, 793-801.

Steefel, C.I., D. DePaolo, and P.C. Lichtner (2005), Reactive transport modeling: An essential tool and a new research approach for the Earth sciences, Earth Planet. Sci. Lett., 240, 539-558, doi: 10.1016/j.eps1.2005.09.017.

Tartakovsky, A. M., G. D. Tartakovsky, and T. D. Scheibe (2009), Effectsof incomplete mixing on multicomponent reactive transport, Adv. Water Resour., 32, 1674-1679.

Tehseen, N., and P. Broadbridge (2012), Fourth order diffusion equations with increasing entropy, Entropy, 14, 1127-1139, doi: 10.3390/e14071127.

Weeks, S. W., and G. Sposito (1998), Mixing and stretching efficiency in steady and unsteady groundwater flows, Water Resour. Res., 34(12), 3315-3322, doi:10.1029/98WR02535.

Weiss, J. (1991), The dynamics of enstrophy transfer in two-dimensional hydrodynamics, Physica D, 48, 273-294, doi: 10.1016/0167-2789(91)90088-Q.

Ye, Y., G. Chiogna, O.A. Cirpka, P. Grathwohl, and M. Rolle (2015a), Influence of flow dimensionality on plume dilution: 2-D vs. 3-D flow-through experiments, J. Contam. Hydrol., 172: 33-47. DOI: 10.1016/j.jconhyd.2014.11.002.

Ye, Y., G. Chiogna, O.A. Cirpka, P. Grathwohl, and M. Rolle (2015b), Experimental evidence of helical flow in porous media, Phys. Rev. Lett., 115 (19), doi: 10.1103/PhysRevLett.115.194502.

Yoon, H., A.J. Valocchi, C.J. Werth, and T. Dewers (2012), Pore-scale simulation of mixing induced calcium carbonate precipitation and dissolution in a microfluidic pore network, Water Resources Research, 48, W02524, doi:10.1029/2011WR011192, 2012. 
Figure 1.
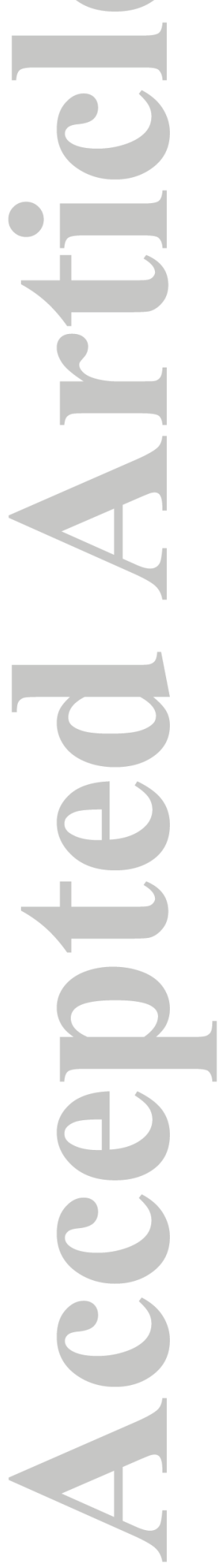

This article is protected by copyright. All rights reserved. 


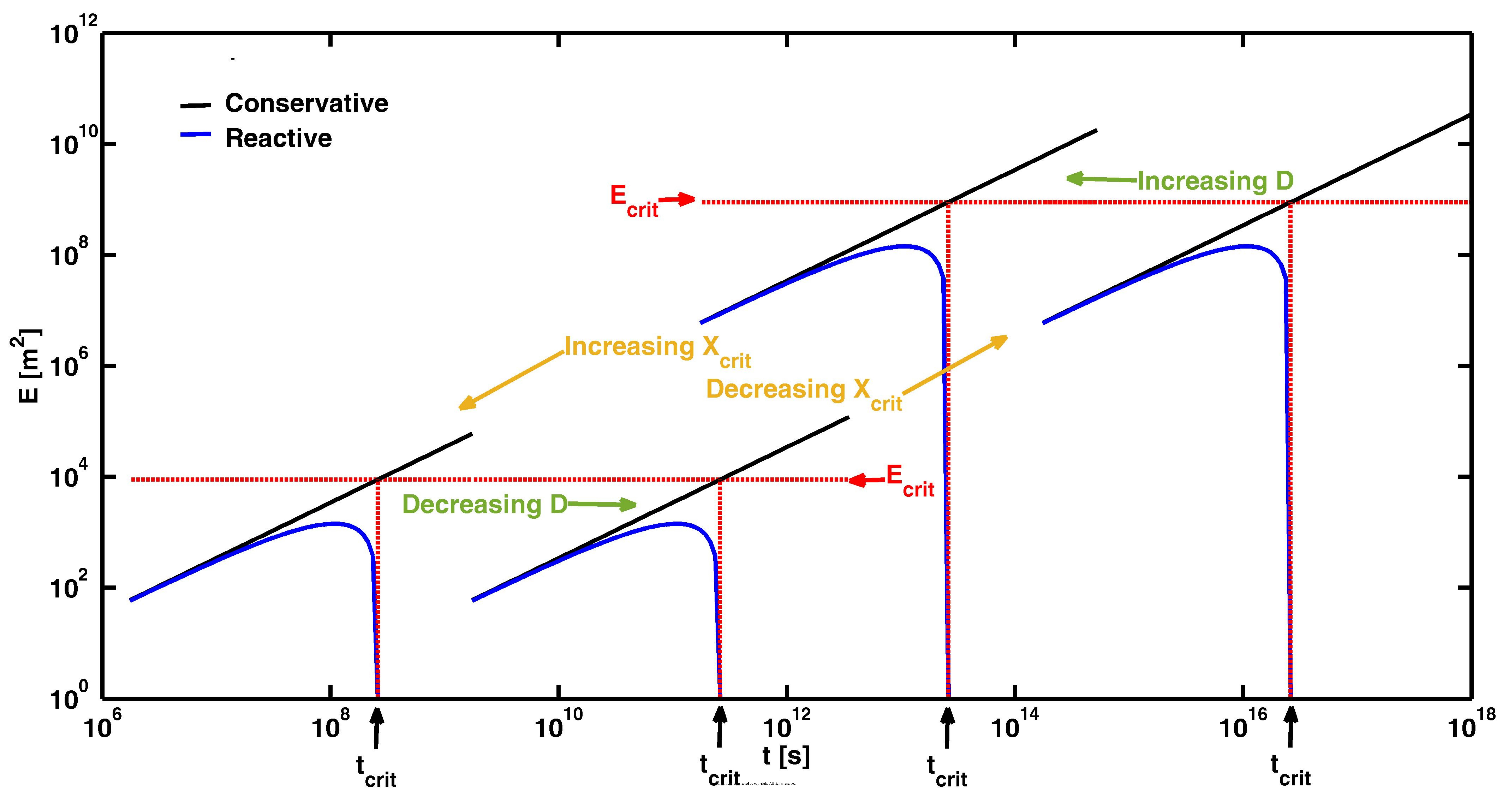


Figure 2.
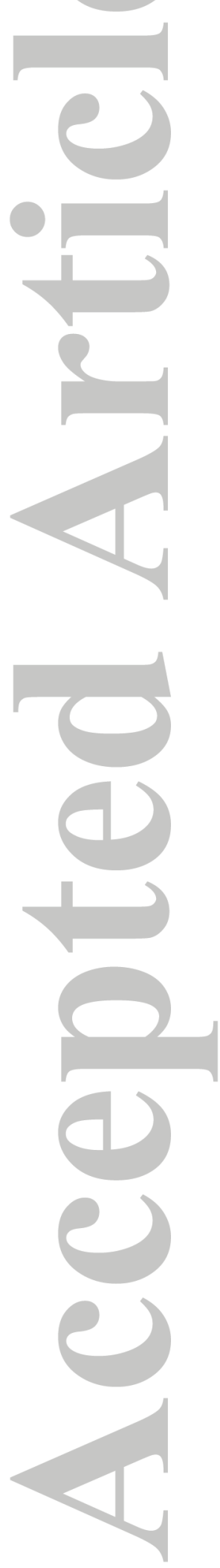

This article is protected by copyright. All rights reserved. 

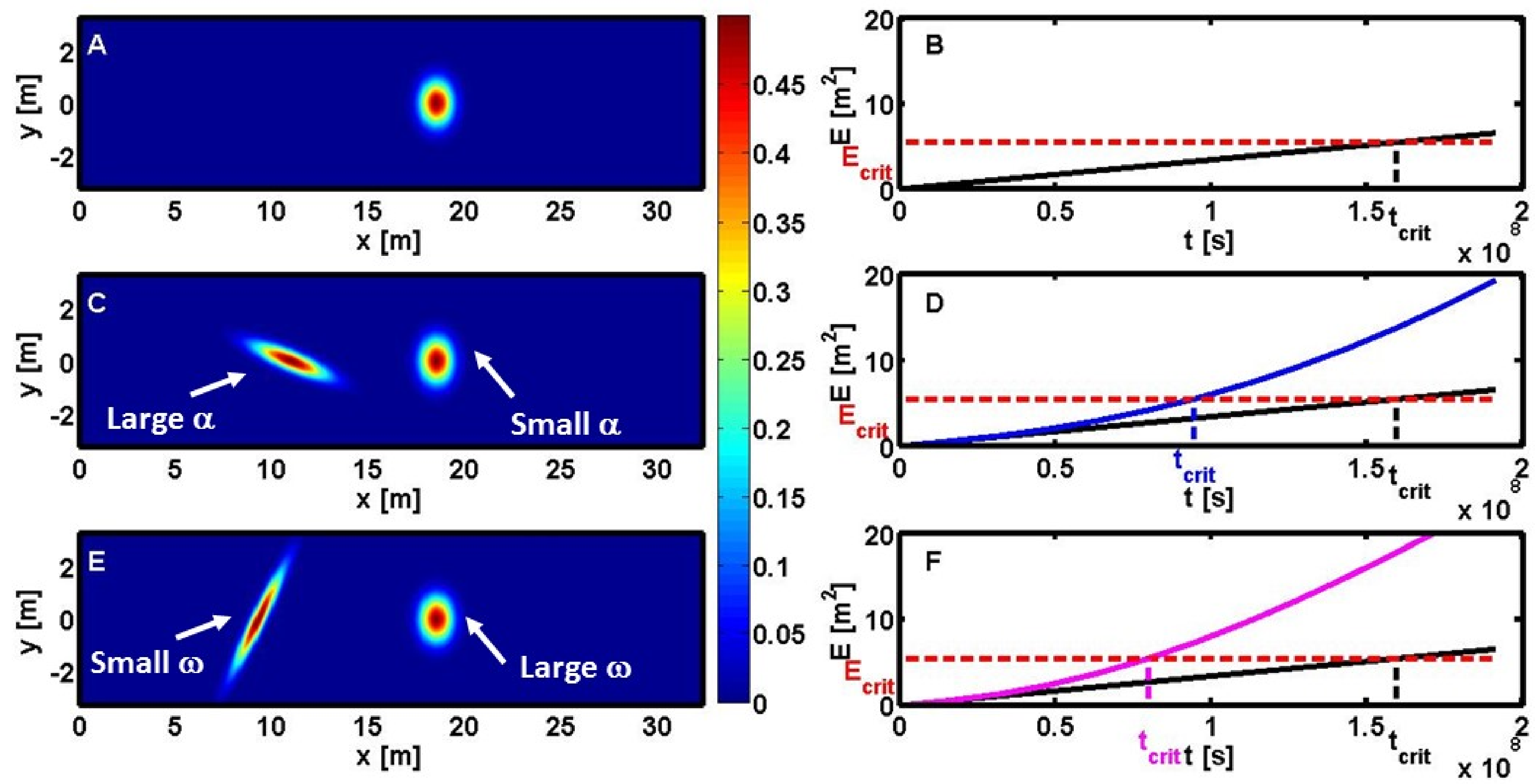
Figure 3.
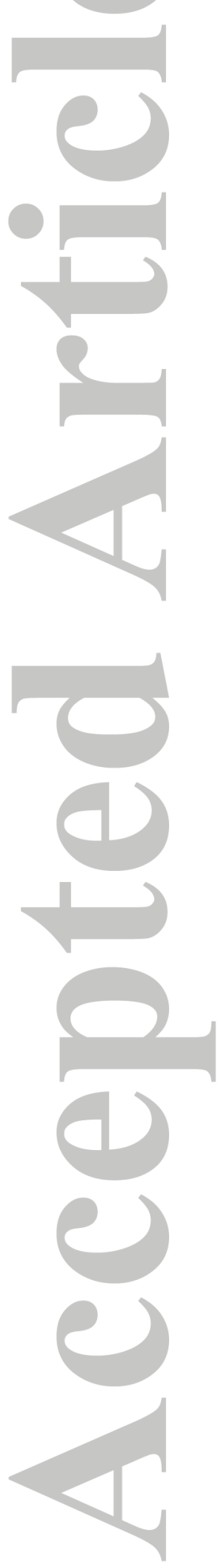

This article is protected by copyright. All rights reserved. 

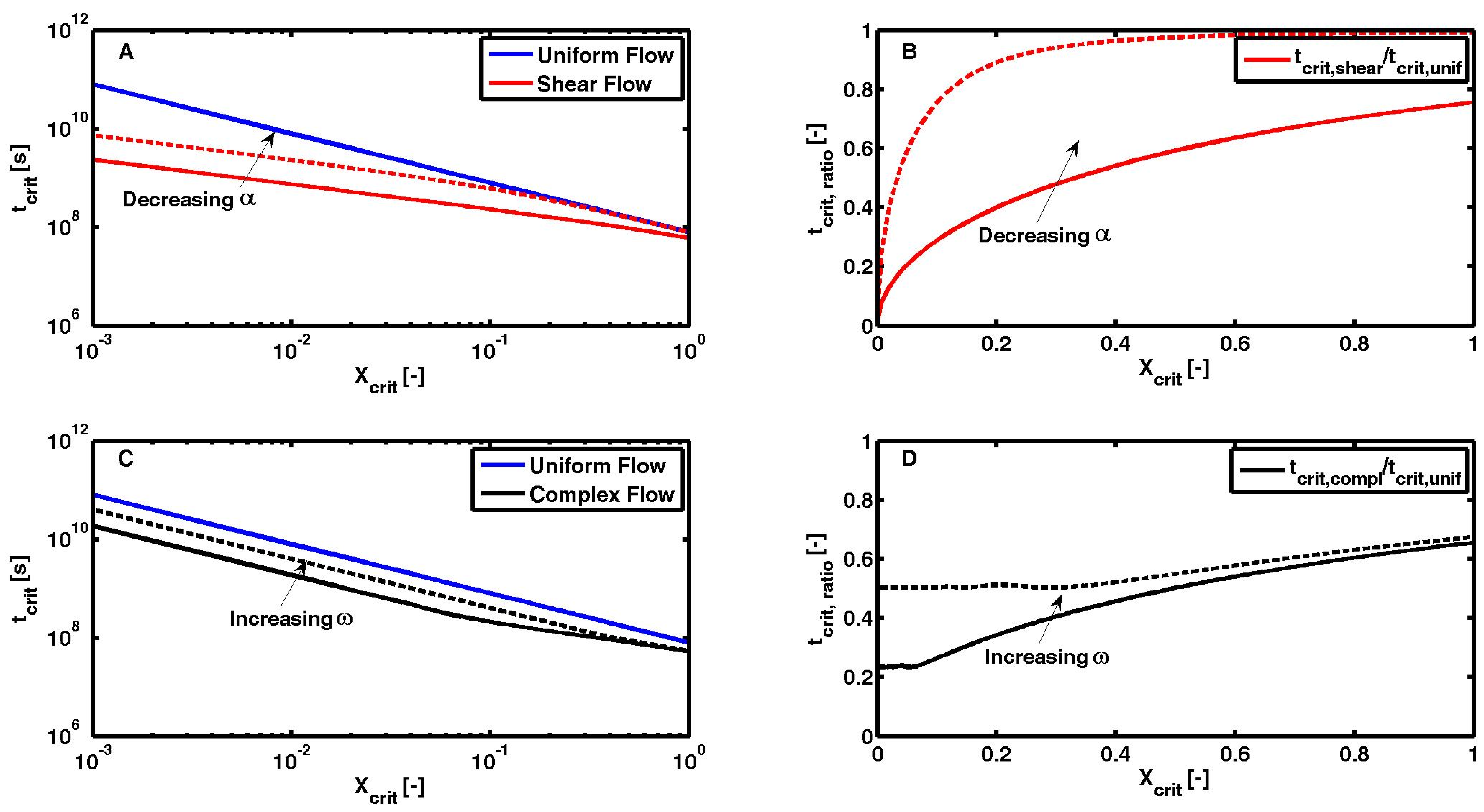